Relations industrielles

Industrial Relations

\title{
Productivité du travail, source de richesse
}

Résumé d'un cours donné par M. Gérard Tremblay (Session intensive-1946)

Volume 1, numéro 9, mai 1946

URI : https://id.erudit.org/iderudit/1023957ar

DOI : https://doi.org/10.7202/1023957ar

Aller au sommaire du numéro

Éditeur(s)

Département des relations industrielles de l’Université Laval

ISSN

0034-379X (imprimé)

1703-8138 (numérique)

Découvrir la revue

Citer cet article

(1946). Productivité du travail, source de richesse : résumé d'un cours donné par M. Gérard Tremblay (Session intensive-1946). Relations industrielles / Industrial Relations, 1(9), 7-7. https://doi.org/10.7202/1023957ar
Résumé de l'article

Résumé d'un cours donné par M. Gérard Tremblay (Session intensive-1946)
Tous droits réservés @ C Département des relations industrielles de l’Université Laval, 1946
Ce document est protégé par la loi sur le droit d'auteur. L’utilisation des services d'Érudit (y compris la reproduction) est assujettie à sa politique d'utilisation que vous pouvez consulter en ligne.

https://apropos.erudit.org/fr/usagers/politique-dutilisation/ 


\title{
PRODUCTIVITE DU TRAVAIL, SOURCE DE RICHESSES
}

\author{
Résumé d'un cours donné par M. Gérard Tremblay \\ (Session intensive-1946)
}

" L'économie politique a pour objets, parmi les rapports des hommes vivant en société, ceux-là seulement qui tendent à la satisfaction de leurs besoins matériels, à tout ce qui concerne leur bien-être. ") (Charles Gide).

L'étude de cette science comprend généralement quatre divisions classiques :

$1^{\circ}$ la production des richesses et l'étude de ses facteurs - nature, capital, travail ;

$2^{\circ}$ la circulation des richesses ;

$3^{\circ}$ la distribution des richesses, part du capital ou profit, part du travail ou salaire ;

$4^{\circ}$ la consommation des richesses.

Le titre de cette causerie nous amène à considérer certains aspects de deux des quatre grandes divisions de l'économie politique :

a) la production des richesses ;

b) la distribution des richesses.

La distribution des richesses est en fonction de leur production. En d'autres termes, le profit et le salaire dépendent des facteurs de la production - nature, capital, travail. Par voie de conséquence, plus les facteurs opèrent avec efficacité, plus abondante est la richesse produite et plus abondants sont le profit et le salaire.

Comment organiser l'efficacité des facteurs de la production? En d'autres termes, est-il possible de rationaliser les trois éléments ou facteurs de la production nature, capital, travail ? Nous répondons dans l'affirmative.

a) Rationalisation de ce qui touche à la nature. L'industrie doit avoir en abondance les matières premières à ouvrer ; celles-ci doivent être de qualité. Les sources de force motrice doivent être en disponibilité.

b) Le capital, qu'il s'agisse du capital immobilisé ou du capital-argent, doit être suffisant. Le capital immobilisé est représenté par l'emplacement, les usines, l'outillage. Le capital-argent est représenté par la finance et les capitaux de roulement.

c) Le travail, facteur principal et essentiel de la production, est représenté par :

$1^{\circ}$ La direction ou la maîtrise ;

$2^{\circ}$ Les cadres : techniciens et contremaitres ;

$3^{\circ}$ Les ouvriers de la production, qui doivent être qualifiés et nombreux.

En faisant donner plein rendement aux trois facteurs ci-haut mentionnés, on assure une production abondante, de haute qualité et à bon marché.

***

Il nous intéresse précisément de savoir comment rationaliser la production du facteur " travail des ouvriers ". Trois éléments y contribuent :

$1^{\circ}$ Les qualifications de l'ouvrier. Celui-ci doit avoir fait un apprentissage sérieux adapté à son métier. Il doit posséder un entraînement professionnel tenant compte de la nature de l'industrie. Il doit être dans des conditions physiques optima; avoir un moral élevé, un esprit compréhensif ; être prêt à collaborer avec les cadres et la mâtrise, en un mot, avoir l'esprit d'équipe. $\mathscr{Z}^{\circ}$ Les cadres ou les techniciens peuvent grandement contribuer à l'accroissement de la productivité de l'ouvrer en ce qu'ils ont la responsabilité de la division du travail qui est nécessaire pour assurer le succès d'une grande entreprise. Cette division du travail entraîne à son tour l'analyse des tâches; leur évaluation; les études chronométrées; les normes de production; le tempo ou la cadence du travail.

$S^{\circ}$ Enfin, la maîtrise, en collaboration avec les cadres et le travail lui-même, peut accroître la productivité en organisant le système de prime à l'économie et de prime au rendement.

La prime peut récompenser les économies directes si elle prévient le gaspillage des matières premières, du temps, de l'outillage et des machines, des matières de frais généraux comme la force motrice, l'éclairage, le combustible. On peut également accorder des primes pour les économies indirectes. Exemple : primes d'ancienneté, primes de régularité en vue de combattre l'absence de l'usine (absenteeism).

La prime au rendement peut s'appliquer au travail à l'heure comme au travail à la pièce. La rémunération au rendement a pour but la stimulation de l'activité par rapport à une production moyenne et normale dans l'unité de temps.

La mise en œuvre des moyens indiqués pour améliorer la productivité de l'ouvrier produit trois effets :

a) augmentation des profits ;

b) élévation des salaires ;

c) réduction des prix pour le consommateur.

C'est la constation de ces effets ou conséquences de la productivité qui a amené des économistes à promulguer la loi économique des salaires. Cette loi s'établit comme suit :

"Le taux de salaire est soumis à la loi de la productivité du travail influencée nécessairement par l'intervention de causes extrinsèques. ")

En effet, une bonne loi des salaires doit expliquer comment les salaires sont plus élevés dans un pays que dans un autre, à telle époque par rapport à telle autre, dans tel métier par rapport à tel autre.

Nous nous sommes particulièrement occupé ici de l'aspect économique de la productivité et du salaire qui la récompense. Il ne faut pas oublier que le salaire a un aspect social et moral, car il fait vivre un être humain dans le cadre réaliste de la vie. Sous cet aspect, il doit être juste et raisonnable et sa fixation doit être guidée par les critères suivants : coût de la vie d'une famille ; situation de l'entreprise ; bien commun.

Il est certain que le social et l'économique se heurtent et s'entrechoquent. On peut les concilier par l'augmentation de la productivité, en autant que celle-ci ne met pas en péril la santé du travailleur. 